\title{
Preparation and Characterization of Amine Modified Carbon Quantum Dots from Mesoporous Carbon
}

\author{
Zhipeng Sun ${ }^{1, a,{ }^{*}}$, Qian Luo ${ }^{2, b}$ and Wensheng $\operatorname{Ran}^{3, c}$ \\ ${ }^{1}$ Xingjiang Uygur Autonomous Region Product Quality Supervison and Inspection Institute, Urumqi \\ 830011, China \\ ${ }^{2}$ Xingjiang Uygur Autonomous Region Product Quality Supervison and Inspection Institute, Urumqi \\ 830011, China \\ ${ }^{3}$ Xingjiang Uygur Autonomous Region Product Quality Supervison and Inspection Institute, Urumqi \\ 830011, China \\ asunzhipengxj@gmail.com, ${ }^{\mathrm{b}} 44733937 @ q q . c o m,{ }^{c}$ 924205855@qq.com
}

Keywords: Mesoporous carbon; Nitric acid oxidation method; Carbon quantum dots; Optical properties

Abstract. Amine modified carbon quantum dots were synthesized though a simple nitric acid oxidation method together with a hydrothermal treatment using mesoporous carbon as precursor. The features and properties of product were characterized by fourier transform infrared spectroscopy (FTIR), photoluminescence spectra (PL), High-resolution transmission electron microscopy (HRTEM), UV-visible spectroscopy (UV/vis) and X-ray diffraction (XRD). The results indicated that the GQDs have bright aquamarine with high water solubility, good photo stability.

\section{Introduction}

Carbon-based quantum dots (CQDs), as recently emerging carbon-based materials, are carbon nanoparticles smaller than $10 \mathrm{~nm}$ in size and graphene nanosheets less than $100 \mathrm{~nm}$ in width called carbon quantum dots [1]. As new promising carbon materials, CQDs have great prospective applications in sensing, diagnosis, imaging, photovoltaic devices and photo catalysis in particular in bio-imaging [2-3], due to their excellent properties including unusual fluorescence, low toxicity, good biocompatibility, easy preparation, environmental friendliness, strong resistance to photo degradation and bleaching [4-5]. Especially, the luminescence properties have been received hot research interest.

To date, synthesis methods of CQD mainly involved both chemical methods and physical methods. Different methods have been developed to prepare CQDs consisting of electrochemical approach [6], re-oxidation [7], hydrothermal route [8], thermal treatment [9], microwave/ultrasonic process [10], arc discharge [11], laser ablation method [12], etc. However, most of these methods have some downsides, which mean difficulty to obtain starting materials, tedious process, poor optoelectrical properties or low yield. On the other side, reasonable selection of carbon source is of great importance to property of CQDs. Thus, there is a great need to choose a suitable carbon source and develop a more efficient method to synthesize CQDs. Mesoporous carbon is a new type of porous materials with an approximate aperture of $2 \sim 50 \mathrm{~nm}$. It has been widely used in catalysis, sensors, lithium battery and so on, because of its high specific surface area, uniform pore size, large pore volume, high mechanical stability and conductivity [13]. However, to the best of our knowledge, there are still few reports on the fabrication of CQDs using mesoporous carbon as precursor. Thus, developing a facile, controllable, and scalable technique to prepare GQDs using mesoporous carbon is becoming highly desirable.

Herein, we report a simple route to synthesize fluorescent amine modified carbon quantum dots through nitric acid oxidation method together with hydrothermal treatment using mesoporous carbon and amine water respectively. 


\section{Experimental}

\section{Chemicals}

All chemicals were of analytical grade and were used without further purification. Deionized water was used throughout the experiment.

\section{Preparation of amine modified carbon quantum dots}

CQDs were prepared from mesoporous carbon by refluxed with concentrated nitric acid. In a typical procedure, $0.2 \mathrm{~g}$ dried mesoporous carbon was put into $30 \mathrm{~mL} 7 \mathrm{M} \mathrm{HNO}_{3}$ followed by refluxing for 24 h. After cooling to room temperature, the suspension was centrifuged at $3000 \mathrm{rpm}$ for $30 \mathrm{~min}$ to remove the large particles and obtain a supernatant. The supernatant was heated to evaporate the water and nitric acid and a reddish-brown solid was obtained. Then solid sample was dissolved in 50 $\mathrm{mL} \mathrm{H}_{2} \mathrm{O}$ and then ultrasonicated for $15 \mathrm{~min}$, and further adjusted to about $\mathrm{pH} 8$ with $1 \mathrm{M}$ and $0.1 \mathrm{M}$ ammonia water. The solution was centrifuged for $30 \mathrm{~min}$ under $10000 \mathrm{rpm}$ and then the filtrate was collected and dried at $80{ }^{\circ} \mathrm{C}$ in vacuum oven.

As-prepared dried sample was mixed with $10 \mathrm{~mL}$ ammonia water, and cleaned by centrifugation for $15 \mathrm{~min}$. The mixture was added into a Teflon lined stainless steel autoclave and heated at $180{ }^{\circ} \mathrm{C}$ for 10-12 h. After cooling down to ambient temperature, the supernatant was collected by centrifugation at $12,000 \mathrm{rpm}$ for $30 \mathrm{~min}$. the obtained solid samples was dried at $80{ }^{\circ} \mathrm{C}$ in vacuum oven and labeled as a-CQDs.

\section{Instrumentation}

The crystal structure of the CQDs was characterized by X-ray diffraction (XRD) (Rigaku, MiniFlex600). The HRTEM image was taken using a 2,100 F field emission gun TEM with GQDs directly transferred onto a C-flat TEM grid. Fourier transform infrared spectra (FTIR) were obtained on a FTIR spectrophotometer (BIO-RAD, FTS165). UV/vis spectra were obtained by a UV/vis spectrophotometer (SHIMADZU, UV-2450). All fluorescence spectra were obtained by a spectrofluorometer (HITACHI, F-4500). High-resolution transmission electron microscopy (HRTEM) observation was carried out on a HRTEM JEM-2010FEF instrument.

\section{Results and discussion}

\section{Characterization of a-CQDs}

The structure and chemical composition of the a-CQDs were investigated with FTIR spectroscopy and X-ray diffraction (XRD), respectively. The result of FTIR spectrum in Fig.1a shows that the broad characteristic peak of $-\mathrm{OH}$ at $3445 \mathrm{~cm}^{-1}$, the strong absorption peak of $\mathrm{C}=\mathrm{O}$ at $1661 \mathrm{~cm}^{-1}$, bending vibration peak of $-\mathrm{OH}$ at $1383 \mathrm{~cm}^{-1}$, and the stretching vibration peaks of C-O-C at $1131 \mathrm{~cm}^{-1}$. It was proved that the presence of plentiful carboxylic acid and other oxygen-containing functional groups on the surface of the a-CQDs, which endowed them with high water solubility [5]. The stretching vibration peaks of $\mathrm{N}-\mathrm{H}$ at 1131 and $3445 \mathrm{~cm}^{-1}$ proved the existence of amino-containing functional groups. The X-ray diffraction (XRD) pattern of a-CQDs shows a broader diffraction peak at $2 \theta=24.36^{\circ}$ in Fig. 1 b, corresponding to the graphitic structure [8]. Comparing with the reference standards, the main peak in the a-CQDs pattern was similar to the typical diffractsion peak of amorphous carbon [9]. 

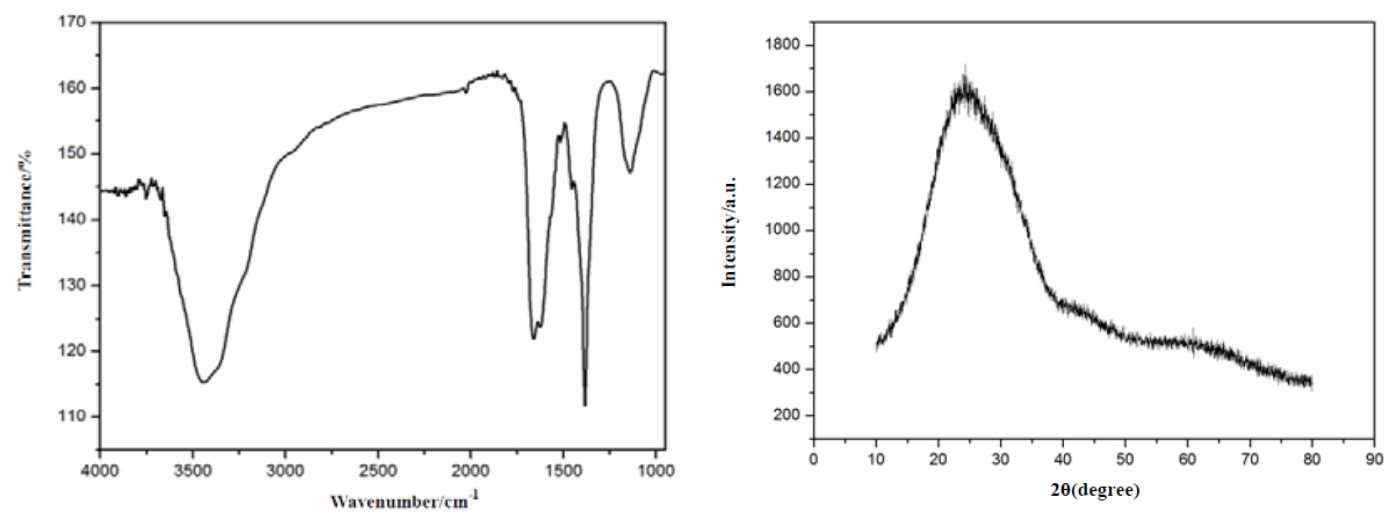

Fig.1 (a) the FTIR spectrum and (b) the XRD patterns of a-CQDs

\section{Optical properties of a-CQDs}

The highly monodispersed property is one of the major advantages of our method over other GODs preparation techniques. The size and mophology of a-GQDs can be seen in Fig. 2a. It can be shown that the a-GQDs are spherical morphologies and uniformly distributed with an average diameter of $5.0 \pm 0.5 \mathrm{~nm}$. The optical properties of the a-CQDs were characterized with UV/vis and PL spectroscopy. As shown in Fig.2b, the absorption spectrum shows a clear absorption peak at $345 \mathrm{~nm}$, it can be seen from Fig.2b inset image that the aqueous solution of a-CQDs was pale yellow and transparent in daylight but changed to a bright aquamarine under UV excitation. The a-CQDs have multicolor fluorescent emissions depending on the excitation wavelength used in Fig.2c. The emission wavelength of a-CQDs was excitation-independent when the excitation wavelength was lower than $440 \mathrm{~nm}$, when the excitation wavelength changed from 440 to $480 \mathrm{~nm}$, the maximum emission peak position also shifted to a longer wavelength, from 506 to $540 \mathrm{~nm}$ (a red-shift of $34 \mathrm{~nm}$ ). As the peak position of maximum emission changed, the PL intensity decreased greatly, showing the clear dependence of emission wavelength/intensity on the excitation wavelength. This phenomenon was similar to that of luminescent carbon nanocrystals (CNCs), and could be explained by the a-CQDs having different sizes and different emissive sites because of different degree of nitric acid oxidation. Narrower size distribution means purer PL and smaller FWHM of PL, which plays an important role in optical properties. On the other hand, it is well known that functionalized graphite particles are hydrophilic, which possibly results from the surface functional groups that located at the interface of the a-GQDs. Thus, our method provides an important, yet very simple approach to tune the a-GQD diameters.
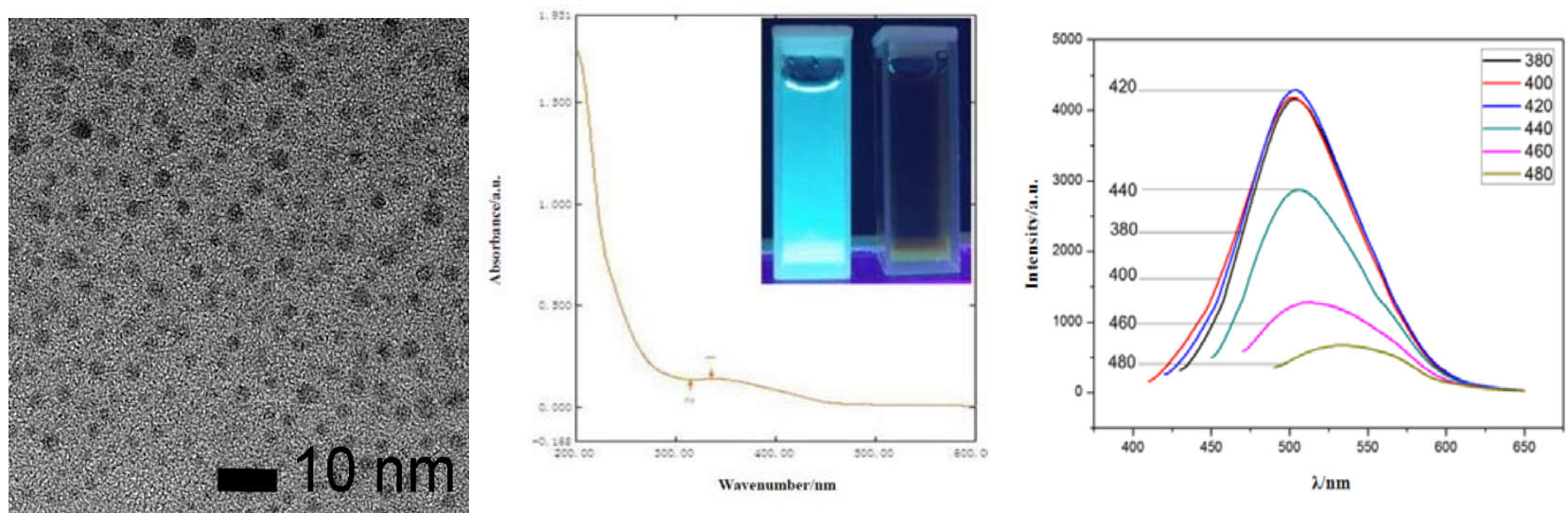

Fig. 2 (a) TEM image of a-CQDs; (b) the UV/vis absorption spectra and (c) photoluminescence emission spectra of the a-CQDs 


\section{Conclusions}

we have demonstrated a simple method to prepare a-CQDs though a simple nitric acid oxidation method together with a hydrothermal treatment using mesoporous carbon as precursor. The results show that the a-CQDs bright aquamarine with high water solubility, good photo stability. The a-CQDs remain to be studied as well as their insertions into optical, electronic and structural composite materials.

\section{Acknowledgements}

This work was financially supported by the Natural Science Foundation of Xinjiang Uygur Autonomous Region (2512211A040).

\section{References}

[1] L.A. Ponomarenko, F. Schedin, M.I. Katsnelson, R. Yang, E.H. Hill, K.S. Novoselov and A.G. Geim: Science Vol. 320 (2008), p. 356

[2] C. Feldmann: Nanoscale Vol. 3 (2011), p. 1947

[3] J.M. Liu, L.P. Lin, X.X. Wang, S.Q. Lin, W.L. Cai, L.H. Zhang and Z.Y. Zheng: Analyst Vol. 137 (2012), p. 2637.

[4] T. Zhu, J.S. Chen and X.W. Lou: J. Phys. Chem. C Vol. 115 (2001), p. 814.

[5] H.P. Liu, T. Ye, C.D. Mao: Angew. Chem. Int. Ed. Vol. 46 (2007), p. 6473

[6] Y. Li, Y. Hu, Y. Zhao, G. Shi, L. Deng, Y. Hou and L. Qu: Adv. Mate. Vol. 23 (2011), p. 776.

[7] Y. Dong, N. Zhou, X. Lin, J. Lin, Y. Chi and G. Chen: Chem. Mater. Vol. 22 (2010), p. 5895.

[8] Y. Sha, J.Y. Lou and S.Z. Bai: Mater. Res. Bull Vol. 48 (2013), p. 1728.

[9] F. Wang, M. Kreiter, B. He, Pang and C.Y. Liu: Chem. Commun. Vol. 45 (2010), p. 3309.

[10] W. Lu, X. Qin, A.M. Asiri, A. Al-Youbi and X.P. Sun: J. Nanopart.Res Vol. 1 (2013), p. 1.

[11] Y. Guo, R.X.Tian, Y.G. Dong and S.L. Hu:Chinese J. Luinescence Vol. 126 (2004), p. 12736.

[12] G. Eda, M. Chhowalla: Adv. Mater. Vol. 22 (2010), p. 2392

[13] J.Y. Pan, M.Y. Shao: Chin. J. Lumin. Vol.32 (2011), p. 277 